\title{
A System of Usability Evaluation of Confocal Microscope Based on Fuzzy Analytic Hierarchy Process

\author{
Yan Zhou ${ }^{1, \text { a }}$, Liang Liu ${ }^{1, b}$
} \\ ${ }^{1}$ Harbin Institute of Technology, Department of Industrial Design, Harbin 150001, China \\ azhouyan@hit.edu.cn, bqiongqiong4822@sina.com
}

\begin{abstract}
As many usability evaluation studies have been conducted in fields of daily-use products, little attention are put on special devices like microscope, surveymeter or other instruments. This paper attempt to establish a system of usability evaluation of microscope through FAHP methods. The usability evaluation indexes of the microscope are classified into software simulation and usability test. The evaluation indexes include efficiency, effectiveness and user satisfaction in the evaluation system. This paper describes an evaluation system and a quantitative method evaluating the usability of microscope visually and effectively.
\end{abstract}

Keywords: FAHP, usability evaluation system, simulation, confocal Microscope.

\section{Introduction}

With the development of economic society and the increasement of people's needs, users are focusing on not only the material comfort but also the mental experience of products. So the usability of the product is particularly important. The objective of this research is to explore a measurement method to make the subjective of user experience quantified to evaluate results objectively. What's more, the significance of this research is to provide decision and special suggestion for enterprise and productivity in design and evaluation of products, especially like the microscopes which belong to high-precision products.

In the field of usability assessment of product, Lindsey N. Clark ${ }^{[1]}$ and his partners study a heuristic method to evaluate an emergency department information system prototype. Deepak Gupta $^{[2]}$ and his partners find that the multistage fuzzy system can make the usability determination more effective. Professor W.B.Lee ${ }^{[3]}$ and his partners demonstrate that furry analytic hierarchy process approach in modular product design can solve many product design problems. Similarly, Yongfeng $\mathrm{Li}^{[4]}$ shows that a product usability evaluation method based on fuzzy analytic hierarchy process.

In view of the above situation of usability evaluation studied, this paper is to build an objective and comprehensive assessment system of microscope to evaluate the user experience through building a comprehensive fuzzy assessment model to quantify the results the overall grade.

\section{Construction of Evaluation System Based on FAHP}

\subsection{Potential Usability Index Collection}

Professional instrument product users, using the environment and the particularity of operation habit, so you cannot refer to general usability. This article selects dimension can be divided into efficiency property, effectiveness, user satisfaction. The literature method to capture and the availability of a 15 potential, as shown in Table 1. 
Table 1 Potential Usability Index Collection

\begin{tabular}{ccccc}
\hline Dimension & Number & Index & Characterization & score \\
\hline \multirow{2}{*}{ Efficiency } & 1 & Hardware Efficiency & Quick Commissioning, Manipulate & 5.14 \\
& 2 & Interface Efficiency & Quick Manipulate & 5.94 \\
\hline & 3 & Logicality & User Habits, Interface Logic & 5.31 \\
& 4 & Layout Reasonable & Comfortable, Convenient, Ergonomic & 5.54 \\
& 5 & Learning Costs & Cheap & 5.68 \\
Effectiveness & 6 & Help file & Detailed Help System & 5.06 \\
& 7 & Find & Convenient & 5.23 \\
& 9 & Suggestion & Key World of Manipulate & 4.88 \\
& 10 & Long-term Memory & Key Position of Button & 4.86 \\
& 11 & Feedback & Mill Operational Process & 5.74 \\
& 12 & Reversibility & Return to The Previous Step, In Time & 5.19 \\
\hline \multirow{2}{*}{ User } & 13 & Aesthetic & Beautiful Interface and Shape & 5.35 \\
satisfaction & 14 & Comfort & Comfortable, Ergonomic & 5.76 \\
& 15 & No indifferent & Friendly & 5.28 \\
\hline
\end{tabular}

The key factor in extracting usability metrics is the operational experience of the target user. There have 15 indicators through the data analysis, to extract the average first six indicators do reference for interface efficiency, comfort, long-term memory, learning costs, layout reasonable and beautiful.

\subsection{The Building of Evaluation System for Usability}

Based on Seffah's usability evaluation system, this paper presents a usability evaluation system for instrument products. The system is divided into four levels, including the dimension layer, the criterion layer, the index layer and the data layer. Will evaluation indexes at all levels coding, coding for 1 class $\mathrm{Ai}(\mathrm{I}=1,2,3 \ldots \mathrm{n})$, 2 levels of encoding for $\mathrm{Bi}(\mathrm{I}=1,2,3 \ldots \mathrm{n})$, tier 3 coding for $\mathrm{Ci}(\mathrm{I}=1$, 2, $3 . . . \mathrm{n})$, as shown in figure 1 to instrument product usability evaluation system. 


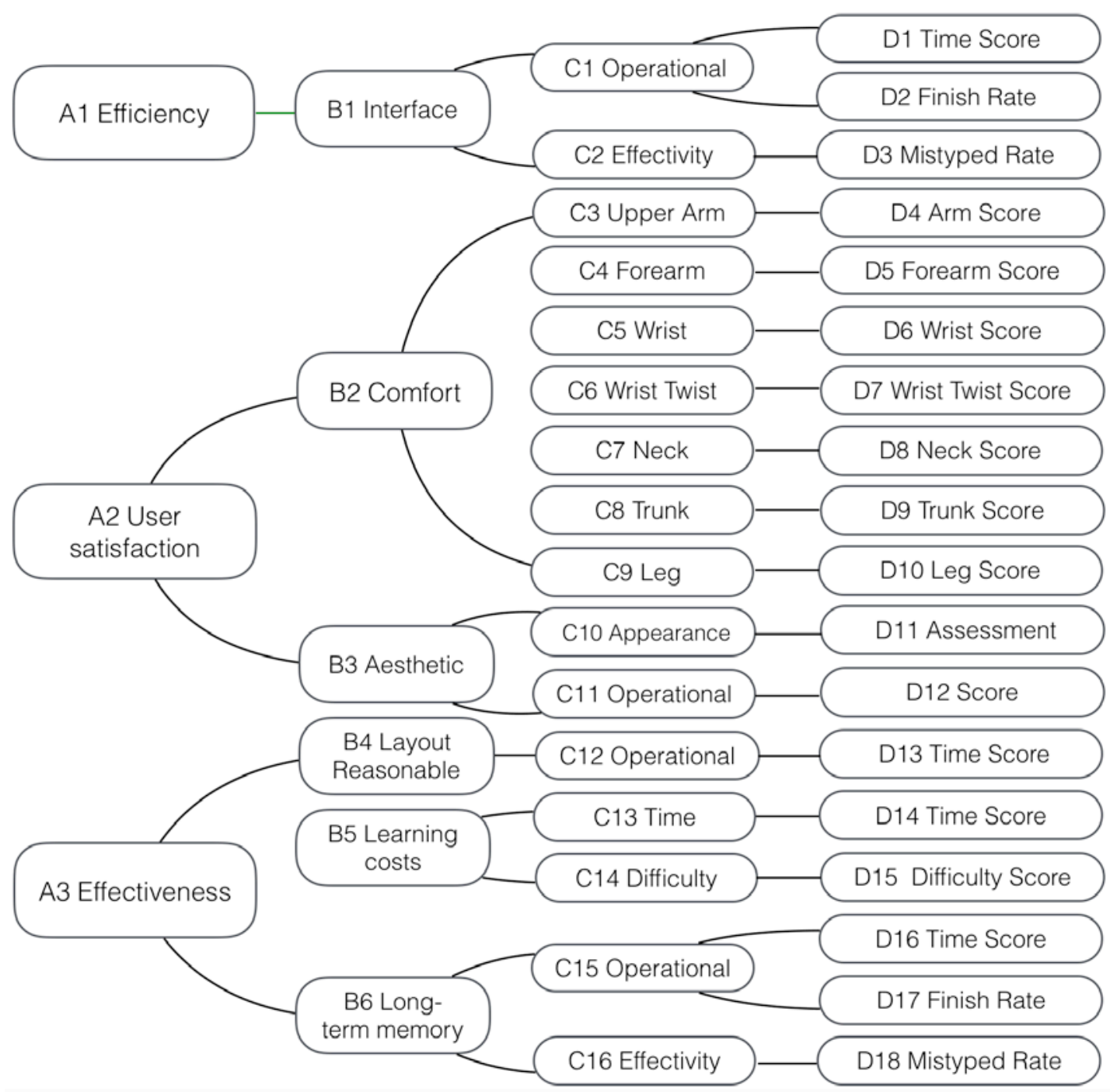

Fig. 1 The usability evaluation system of instrument products

\section{Measures of usability evaluation indicators}

\subsection{Measures of comfort indicators}

The RULA method, which is based on Mc Atamney, evaluates the comfort of the operator. According to the RULA simulation, the simulation operator's operation and operating space of the three schemes are shown in figure 2 .

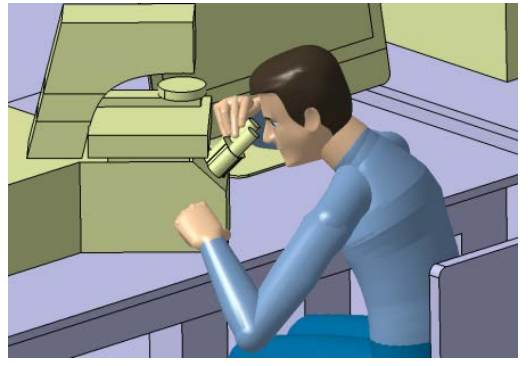

a

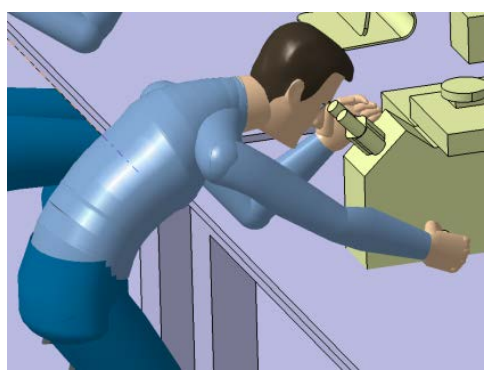

b

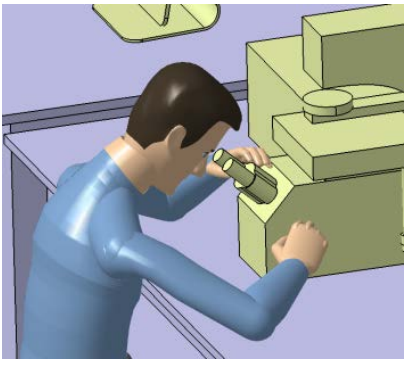

C

Fig. 2 Simulation of the three program

The experiment concluded that plan A had the highest score, indicating that the position of plan A was the most comfortable. In Plan A, virtual people left wrist appeared uncomfortable phenomenon, but in right one, which does not appear the phenomenon of uncomfortable. Plan A right arm wrist comfort index is higher. 
Plan B left arm and forearm and hand joint the whole arm appeared obviously not comfortable, although the sitting position is better than plan $\mathrm{A}$, but the long-time operation will be $\mathrm{A}$ faster than the methods fatigue, therefore, plan B is not better than A.

Plan $\mathrm{C}$ main problems existing in the space for the operation of the eyepiece is unreasonable, virtual people can only be got up and was kneeling posture, leg, knee, ankle under more pressure, long time debugging instrument can quickly lead to fatigue, so this plan comfortable score is relatively low. Arrange the comfort index score for A, B, and C, as shown in table 2.

Table 2 Comfort index scores

\begin{tabular}{|c|c|c|c|c|c|c|}
\hline \multirow{2}{*}{ Position } & \multicolumn{2}{|c|}{ Program A } & \multicolumn{2}{|c|}{ Program B } & \multicolumn{2}{|c|}{ Program C } \\
\hline & Left & Right & Left & Right & Left & Right \\
\hline Upper Arm & 3 & 4 & 4 & 4 & 4 & 4 \\
\hline Forearm & 2 & 2 & 3 & 2 & 1 & 3 \\
\hline Wrist & 3 & 2 & 3 & 3 & 2 & 2 \\
\hline Wrist Trist & 1 & 1 & 1 & 1 & 1 & 1 \\
\hline Neck & \multicolumn{2}{|c|}{4} & \multicolumn{2}{|c|}{1} & 4 & 4 \\
\hline Trunk & \multicolumn{2}{|c|}{3} & \multicolumn{2}{|c|}{2} & 2 & 3 \\
\hline Leg & \multicolumn{2}{|c|}{1} & \multicolumn{2}{|c|}{1} & 1 & 1 \\
\hline Left and Right posture & 3 & 4 & 4 & 3 & 5 & 5 \\
\hline
\end{tabular}

\subsection{Measures of layout rationality indicators}

According to the simulation, the operator's operating distance between the two devices is output, as shown in figure 3 , and data is shown in table 3.

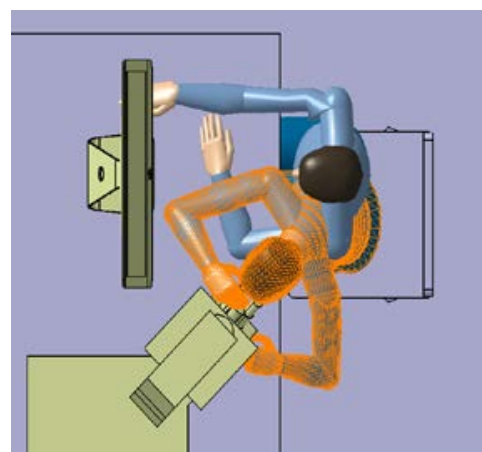

$\mathrm{a}$

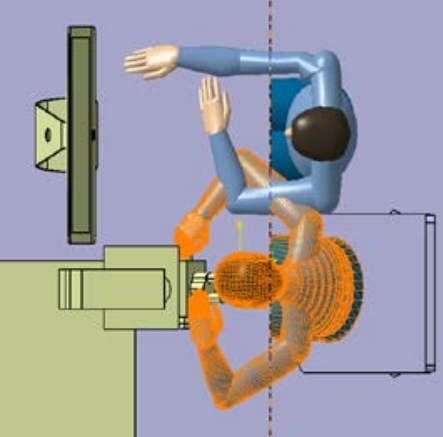

b

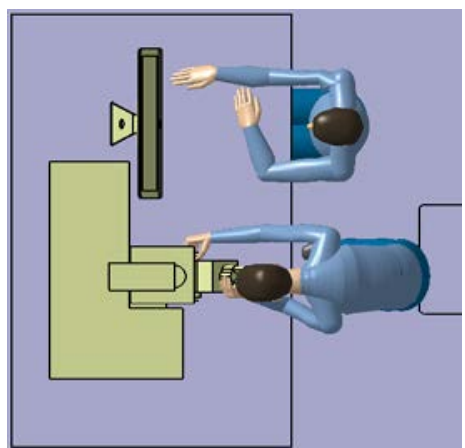

C

Fig. 3 Simulation of the three operating distances

When the operator switches between devices, the length of the moving distance affects the efficiency of the operation, and frequent switching can cause fatigue to the operator. As a result, the shorter the distance, the less likely it will be, the less fatigue will be, and the operational efficiency will be improved.

Table 3 Simulation results

\begin{tabular}{cccc}
\hline & Program A & Program B & Program C \\
\hline Horizontal & 137 & 430 & 674 \\
Vertical & 0 & 0 & 392 \\
Total Displacement & 137 & 430 & 780 \\
\hline
\end{tabular}

\subsection{Measures of Interface evaluation indicators}

The task performed is shown in the table 4 . To experiment the objectivity and accuracy, choosing 12 exposed to complex software but not to use the microscope of the participants, each group a total of three groups of four, and experiment to obtain the data. 
Table 4 Operation task setting

\begin{tabular}{cc}
\hline Task Name & Task Description \\
\hline T1 & Click on Initialization and Live Scan \\
T2 & Select the Laser of 405 and the 535 type of Bandpass Center \\
T3 & Adjust the Laser strength to the strongest \\
T4 & Adjust the Probe Gain to 0.75 \\
T5 & Adjust the Scan Lines to 512 \\
T6 & Set the PZT starting value of 5, and the Endpoint value is 45 \\
T7 & Adjustment Layers to 30 \\
T8 & Confirm the parameters and click the Scan button \\
\hline
\end{tabular}

In the learning cost test, the average learning time for program B is $84.6 \mathrm{~s}$, which is the easiest part of the solution. The average operating time of program B is $24.6 \mathrm{~s}$, the highest efficiency in the experimental data of interface operation efficiency. The long-term memory test data found that the completion rate, the number of errors and the efficiency of program B were the best.

\subsection{Measures of Interface evaluation indicators}

Each time the usability test was completed, the participants are given a beauty questionnaire, and the satisfaction data obtained are shown in table 5.

Table 5 Interface aesthetics score

\begin{tabular}{lcccc}
\hline & Learning costs & interfacial efficiency & Long-term memory & average score \\
\hline Program A & 5.2 & 4.5 & 4.6 & 4.8 \\
Program B & 6.1 & 6.8 & 7.3 & 6.7 \\
Program C & 6.8 & 6.9 & 7.2 & 6.95 \\
\hline
\end{tabular}

\section{Results of overall}

FAHP is a multicriterion decision method for quantitative analysis of qualitative issues, which uses fuzzy numbers to denote the degree of cognitive strength of a person. According to algorithm of number, let triangular fuzzy number $\tilde{A}=\left(\mathrm{a}_{1}, \mathrm{a}_{2}, \mathrm{a}_{3}\right), \widetilde{B}=\left(\mathrm{b}_{1}, \mathrm{~b}_{2}, \mathrm{~b}_{3}\right)$.

$\tilde{A} \oplus \tilde{B}=\left(\mathrm{a}_{1}+\mathrm{b}_{1}, \mathrm{a}_{2}+\mathrm{b}_{2}, \mathrm{a}_{3}+\mathrm{b}_{3}\right)$

$\tilde{A} \otimes \tilde{B}=\left(\mathrm{a}_{1} * \mathrm{~b}_{1}, \mathrm{a}_{2} * \mathrm{~b}_{2}, \mathrm{a}_{3} * \mathrm{~b}_{3}\right)$

Fuzzy assessment matrix $\mathrm{A}=\left(\widetilde{a_{\mathrm{l}}}\right)_{m * \mathrm{n}}$, fuzzy weight vectors $\mathrm{W}=\left(\widetilde{w_{\mathrm{J}}}\right)_{1 * \mathrm{n}}$, and R stands for fraction vector, then we can get:

$R=A \otimes W^{T}=$

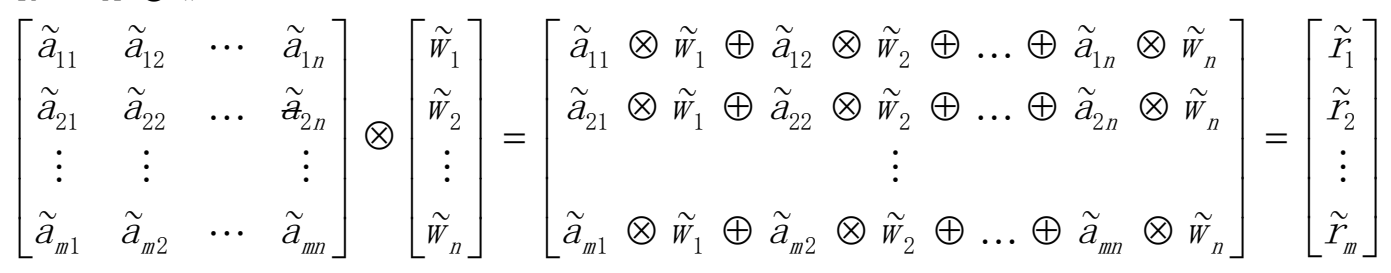

Triangular fuzzy number $\tilde{r}_{i}(1, \mathrm{~m}, \mathrm{u})$, the average $\bar{X}\left(\tilde{r}_{i}\right)$ and standard deviation $\sigma\left(\tilde{r}_{i}\right)$, then we can get:

$\bar{x}\left(\widetilde{r}_{l}\right)=\frac{1}{4}(l+2 m+u)$

$\bar{\sigma}\left(\widetilde{r}_{l}\right)=\frac{1}{80}\left(3 l^{2}+4 m^{2}+3 u^{2}-4 l m-2 l u-4 m u\right)$

At last, fraction vector of user experience is dealt by defuzzification and user experience of three program is ordered in accordance with triangular fuzzy ordering rule, as shown in Table6. 
Table 6 Results of Overall Rating

\begin{tabular}{ccccccc}
\hline \multirow{2}{*}{ Rating } & \multirow{2}{*}{ Samples } & \multicolumn{3}{c}{ triangular fuzzy parameters } & \multicolumn{2}{c}{ defuzzification } \\
& & $l$ & $m$ & $\mu$ & $x$ & $\sigma$ \\
\hline 1 & Program A & 44 & 70 & 106 & 72.50 & 97.35 \\
2 & Program B & 42 & 62 & 102 & 67.00 & 95.00 \\
3 & Program C & 39 & 59 & 97 & 63.50 & 88.15 \\
\hline
\end{tabular}

\section{Conclusions}

On the basis of FAHP theory, the study describes appearance perception, interface perception and user satisfaction as evaluation. Thus, the evaluation system of user experience can be established. Proper measurements are chosen to quantify in accordance with various characteristics of every index through building usability assessment system. The integrated scores can be achieved to assess the user experience of microscope. The paper probes the establishment of evaluation system on usability and quantitative methods in line with the example of microscope. It is also applicable for the quantitative methods and evaluation system of other instrument and equipment.

\section{References}

[1] Lindsey N. Clark, Natalie C. Benda, Sudeep Hegde. "Usability evaluation of an emergency department information system prototype designed using cognitive systems engineering techniques,” Applied Ergonomics, Volume 60, pp. 356-365, April 2017.

[2] Deepak Gupta, Anil K. Ahlawat. "Usability Determination Using Multistage Fuzzy System," Procedia Computer Science, Volume 78, pp. 263-270, 2016.

[3] W.B.Lee, Henry Lau, Zhuo-zhi Liu, Tam. "A fuzzy analytic hierarchy process approach in modular product design,” IEEE Trans. Expert Systems, vol.18,no.1, pp.32-42, Feb. 2001.

[4] Yongfeng Li. "Product usability evaluation method based on fuzzy analytic hierarchy process," Journal of Mechanical Engineering, vol.48, no.14,pp. 183-191, July. 2012.

[5] G.C. Cupchik, Emotion and Industrial Design: Reconciling Means and Feelings, in Proceedings of the 1 st International Conference on Design and Emotion, pp. 75-82, 1999.

[6] M. Baxter, Product Design: A Practical Guide to Systematic Methods of New Product Development. Chapman \& Hall, 1995.

[7] Pengxiang Jin, Chao Hu, Weixing Liu. "Interface design for multi-parameter health monitoring instrument based on ARM-9 embedded system” IEEE International Conference on Information \& Automation, pp. 908-913, 2014. 